\title{
'Face time' for the first time: Video communication between relatives and junior doctors in the COVID-19 pandemic
}

\author{
Authors: Harriet L White, ${ }^{\mathrm{A}}$ Alexander A Tuck, ${ }^{\mathrm{B}}$ Benjamin C Pyrke, ${ }^{\mathrm{B}}$ Emily Murphy, ${ }^{\mathrm{C}}$ Katherine Figg, ${ }^{\mathrm{C}}$ \\ Gwendolen J Cartwright, ${ }^{C}$ Badr A Abdalla ${ }^{\mathrm{D}}$ and Mark A Reynolds ${ }^{\mathrm{E}}$
}

\begin{abstract}
Working on a COVID-19 ward presents a number of challenges to staff, with communication between families, patients and staff being highlighted as a key challenge. Novel methods are needed to overcome the barriers presented by COVID-19, with many turning to technology to offer solutions. Recognising these challenges as an area for improvement on our own ward, we sought to introduce new methods of communication to improve patient, relative and staff understanding and wellbeing. Through our own experiences and a discussion of the literature on this topic, we have identified some key themes which we believe can assist in the development of communication strategies in the developing pandemic.
\end{abstract}

KEYWORDS: COVID-19, video communication, quality improvement, junior doctors, communication

DOI: 10.7861/clinmed.2020-0925

\section{Introduction}

On 11 March 2020, a global pandemic was declared by the World Health Organization, triggering global action to contain, delay, mitigate and research the spread of the novel coronavirus, SARS-CoV-2. Throughout the first wave of the COVID-19 pandemic, supporting effective communication between patients and their families was a recognised challenge for healthcare professionals. ${ }^{1,2}$ Social distancing and hospital visiting restrictions were necessary interventions to mitigate viral spread. This impeded clinicians' ability to deliver family-centred care. This is a concept which has been highlighted in the literature as important in preparing family members for decision making and care-giving

Authors: Afoundation year-2 doctor, University Hospital Llandough, Llandough, UK; ${ }^{B}$ senior house officer, University Hospital Llandough, Llandough, UK; C foundation year-1 doctor, University Hospital Llandough, Llandough, UK; ${ }^{\mathrm{D}}$ Clinical fellow gastroenterology, University Hospital Llandough, Llandough,

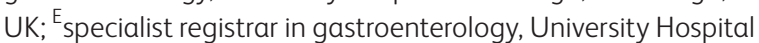
Llandough, Llandough, UK roles, respecting current care-giving roles, reducing family member anxiety, enabling nominated care-givers to advocate for patients and maintaining family integrity. ${ }^{3}$

A large prospective observational study published in the $B M]$ studying the characteristics and outcomes of 20,133 patients reported that the vast majority ( $83 \%$ ) of inpatients admitted with COVID-19 received ward-level care only, with a mortality rate of $26 \%$ within this cohort. ${ }^{4}$ In end-of-life advanced care planning, a family-centred approach is essential to facilitate relative involvement in discussions around death and allow relatives to say goodbye. ${ }^{3}$ This has been shown to be helpful in the grieving process for bereaved family members. ${ }^{5}$ Facilitating communication via telecommunication and video-calling at a ward level is, therefore, more important than ever. However, with minimal clinician education, preparation and relevant technological infrastructure available, this has presented a challenge to effectively implement.

There has been a multitude of articles on video and telecommunication in the outpatient setting throughout the COVID-19 pandemic so far. These have explored the advantages and disadvantages of online consultant or nurse-led clinics, or of general practitioner (GP) phone triaging systems. ${ }^{6-8}$ There is also a large body of literature suggesting models for good communication with relatives in those critically unwell and on intensive care units. ${ }^{1,2}$ However, as junior doctors working on a medical ward amid the initial COVID-19 wave, we found there was very little guidance available on how to initiate a successful communication strategy. Therefore, we developed a quality improvement project with the aim to improve the communication experience of patients and their relatives by implementing video and telecommunication strategies. The project took place during May 2020, during the initial wave of the pandemic. As we continue to manage local outbreaks throughout the UK, this data is more relevant than ever to guide future communication strategies and to learn from our previous experience.

\section{Methodology and results}

We initially interviewed, via telephone, 22 nominated next of kin relatives of patients admitted to a COVID-19 ward in the University Hospital Llandough during May 2020. The purpose of the call was explained, and each respondent gave verbal 
Fig 1. Concerns expressed by relatives in the context of their inability to visit. The values in brackets denote the number and percentage of relatives expressing each concern out of the 22 interviewed.

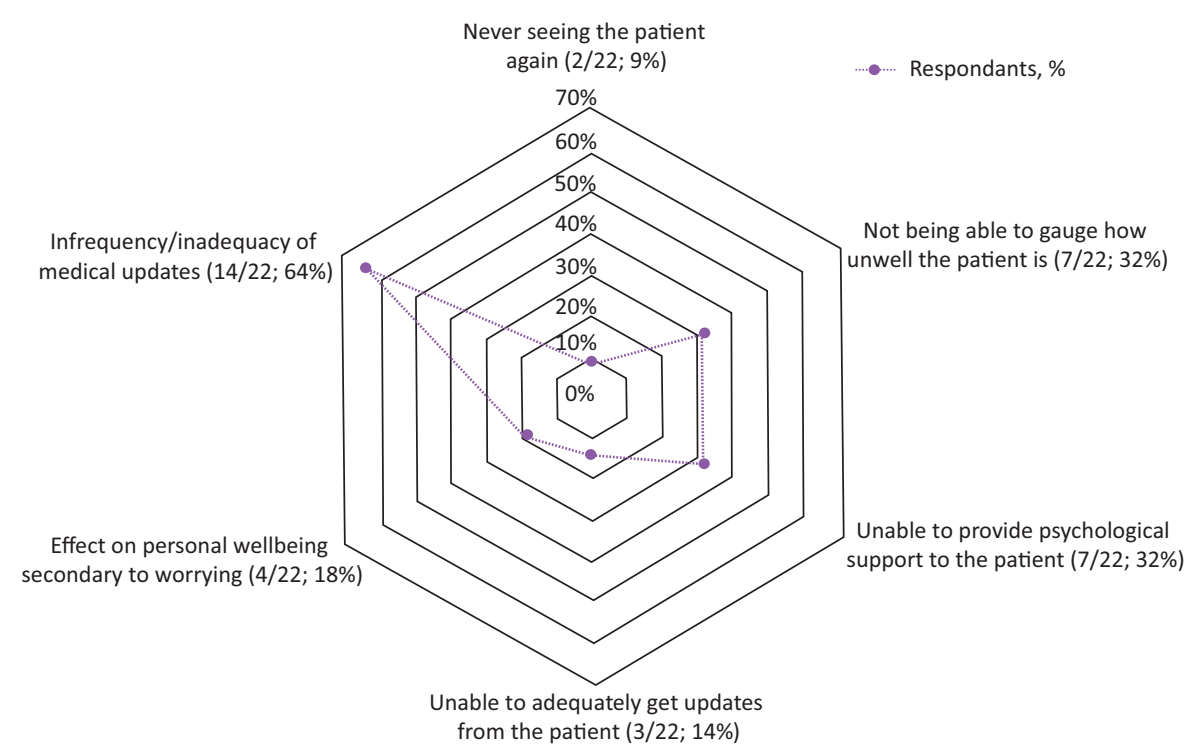

consent for their responses to be documented and anonymised. Their responses were recorded using standardised questions (supplementary material S1) and the results extracted.

We then provided relatives with the opportunity to engage with 20 minute video call updates from a doctor, with patient consent. On calling the ward, relatives were encouraged to book an appointment for a video call update with a doctor. In appropriate scenarios, we used video calling to break bad news and to facilitate video calls between patients and their relatives, sometimes as patients were nearing the end of their life. Qualitative feedback from family members was recorded following these sessions. These video calls were conducted via Accurx, an NHS approved video communication platform. The project was approved by the Cardiff and Vale University Health Board head of quality and safety.

From our initial survey results, $63 \%$ of respondents reported not feeling well informed around their relatives' admission. Key themes were identified from the qualitative feedback we received (Fig 1).

Relatives' most commonly expressed concern was regarding the infrequency or inadequacy of medical updates. Within this, relatives described not being able to get a response when calling the ward, not gaining adequate medical information from updates from other members of the multidisciplinary team (MDT) and struggling to gauge how their relative is doing based solely on verbal updates.

After the implementation of regular video calls, relatives reported increased satisfaction around the communication they had received. They had an improved understanding around the current clinical plan, the events of the admission and felt reassured by the quality of care that their relative was receiving. When using video calls to break bad news, the use of technology enabled a family member to be involved in the discussion, to receive information at the same time as the patient and to provide emotional support. In end-of-life care, 'video visiting' enabled families to say goodbye to their loved ones and to come to terms with their deterioration. One relative reflected that 'seeing their loved one unwell, unresponsive but appearing comfortable and cared for' was a positive experience. The time per phone call was experimentally set at 20 minutes, however, the actual length of video calls ranged from approximately 5 to 30 minutes depending on the amount and detail of information in the discussion and the relatives' level of engagement.

Our study was limited by the quick turnover of patients on the ward, which meant that there were some relatives we were unable to follow up. The nature of the conversations that took place over video call, often involving an element of breaking bad news or discussion around end-of-life care meant that we did not feel it was appropriate to follow up with a rigid questionnaire. The post-intervention results were, therefore, qualitative comments collected based on feedback received following these video calls. We did, however, identify some key themes, which are congruent with themes identified in the literature that we believe can assist in the development of communication strategies in the future.

\section{Discussion}

Our initial survey results suggested that the majority of relatives felt that their communication needs were not being adequately met during the COVID-19 pandemic. This was largely due to infrequency of communication and the perceived difficulty in effectively gathering information over the phone. The inability to visit relatives in hospital not only impacts relatives emotional wellbeing by compromising their role as caregivers, but also restricts family face-to-face exposure to medical professionals. ${ }^{3}$ Published American guidance recommends that families are contacted by a doctor as soon as the patient is transferred or admitted. At this point, the best contact should be identified and a communication plan clearly documented in the notes. Family members should be updated regularly, and clinical teams should strive to provide consistency of communication to develop a good rapport and establish trust. ${ }^{3}$

A lack of non-verbal communication was highlighted during our survey as being one of the greatest challenges during telephone communication. It is well recognised that non-verbal communication, body language and non-verbal cues are key elements of human interaction and can be particularly important 
in the process of breaking bad news. ${ }^{9}$ Non-verbal communication enables us to adjust the way we communicate by being receptive to facial expressions and body language. ${ }^{10}$ We found that video communication enabled an element of non-verbal communication to be retained. This meant that family members received non-verbal cues from the medical professional providing the information and allowed them to visually gauge how their relative was doing.

Choe et al also found that time was an important element, and that having a responsive, active listener take the time to explain and answer their questions was much more preferable than being given a stream of information. ${ }^{10}$ In this study, the allocated slots were 20 minutes in length but, in practice, the time spent on the video call ranged substantially depending on the individual conversation. Time is not only a key element within individual consultations but also achieves a cumulative effect, meaning a rapport is built up throughout multiple discussions. Evidence has shown that breaking bad news conversations are often interpreted on a background of the previous relationships they had built with a doctor, regardless of how the difficult conversation itself went. ${ }^{11}$ This consolidates the importance of regular family updates, ideally with the same doctor if possible and building rapport prior to a potential deterioration. During the COVID-19 era, healthcare professionals have been working around the clock to deliver the best care possible for their patients, and family updates often fall towards the bottom of the priority list behind more clinically crucial tasks. It is, therefore, paramount that introductions and regular updates to relatives are built into daily ward procedure to ensure that this important element of patient care is not missed. We suggest a standardised time of 20 minutes allows for some flexibility. Those leading the video calls may choose to use their own judgement on this, as some conversations are likely to be shorter than 20 minutes with discussions that are predicted to take longer perhaps occupying a double-booking slot.

These recommendations mirror those of the National Healthcare Communication Programme, who have produced specific COVID-19 guidance. ${ }^{12}$ One of the key themes that is consistent throughout is the importance of preparation when undertaking these conversations. This enables clinicians to approach the conversation with a clear focus, answer questions effectively and to support families through this difficult time. Doctors working in the Chelsea and Westminster Hospital NHS Foundation Trust have released a useful infographic to act as a prompt when having these difficult conversations with relatives. ${ }^{13}$ These could be usefully implemented to reduce clinician anxiety around challenging discussions, and provide a structured approach without automating the process or losing the human element.

In end-of-life care, we received positive feedback when video calling was used to facilitate virtual visiting to say goodbye. Enabling meaningful communication between patients and their families has been shown to improve post-bereavement outcomes. Otani et al suggest that having the opportunity for meaningful communication prior to death may be even more important than being present at the time of death. ${ }^{14}$ In the context of this study, this might suggest that by facilitating communication at an earlier stage, via video communication, we can optimise the chance of the patient having a 'good death' by enabling family members to say goodbye. Ultimately, this has the potential to reduce the risk of complicated grief and depression in relatives during bereavement. ${ }^{14}$

Our results also showed that, in some cases, relatives not being able to contact the ward was a significant barrier to communication. Introduction of standardised communication packs and pre-written letters that could be given to families following their relative's admission to hospital could be considered by institutions as a possible solution. This has been suggested by Azouley and Kentish-Barnes in their five-point strategy for improved communication with relatives of patients with COVID-19. ${ }^{15}$ These would provide details of how to access the communication options available, manage relatives' expectations and play a role in a more structured communication pathway. Close involvement of the wider MDT is also recommended for teams aiming to implement video communication strategies. Physiotherapists, occupational therapists, nursing staff and other allied health professionals being present on video will imitate pre-COVID discharge planning meetings and will allow the visual demonstration of mobility, assistance, and care strategies. Video demonstration of mobility or function with the patients (providing adequate consent has been obtained) will also help families understand care needs on discharge.

A significant concern regarding the routine use of video telecommunication is the inability of some families to understand and operate the technology required. Indeed, our study showed that of those who felt video communication was not useful, the predominant reason was anxiety over how to utilise technology. Differing access to internet/telecommunication devices as well as general understanding among relatives and age were found to be factors related to this anxiety. These concerns could be mitigated with the assistance of other family members, however, those relatives who are isolated risk being left behind.

\section{Conclusion}

Good communication with relatives requires time and preparation and, as medical professionals, we should strive to develop a relationship with our patients' relatives regardless of where the patient is on their inpatient journey. Our suggestion to junior doctors is that they take responsibility for building the necessary protocols and pathways to make regular updates and early rapport building a part of daily ward life. We also suggest using video technology where possible to enable face-to-face communication, not just in standard daily medical updates but also in facilitating relatives to talk to their loved ones and in breaking bad news. This will be beneficial to patients and their relatives by supporting carers to remain active in their care-giving roles, improve grief and bereavement outcomes and improve the psychological wellbeing of patients and their families. The burden of this task will decrease with regularity and habit, as we all become used to the 'new normal' within our medical practice.

\section{Key points}

> Communication training should be implemented for foundation level junior doctors who often have the greatest contact time with families but the least experience at delivering difficult news.

> Information on how to access and utilise the video communication technology should be available on the ward and taught during hospital and ward inductions.

> Allocating a specific junior doctor to communicate with families daily would allow them to take ownership of this task and, by providing 'protected time', will improve the regularity of updates and cultivate good rapport. 
> Standardised communication packs that outline protocol should be distributed to patient relatives on admission in order to help align expectations and maintain a structured approach to communication in this uncertain time.

\section{Supplementary material}

Additional supplementary material may be found in the online version of this article at www.rcpjournals.org/clinmedicine: S1 - Family questionnaire.

\section{References}

1 Estella A. Compassionate communication and end-of-life care for critically ill patients with SARS-CoV-2 Infection. J Clin Ethics 2020;31:191

2 Wakam GK, Montgomery JR, Biesterveld BE, Brown CS. Not dying alone - modern compassionate care in the Covid-19 pandemic N Engl ] Med 2020;382:e88.

3 Hart JL, Turnball AE, Oppenhein IM, Courtright KR. Familycentered care during the COVID-19 era. J Pain Symptom Manage 2020;60:e93-7.

4 Docherty AB, Harrison EM, Green CA et al. Features of 20133 UK patients in hospital with covid-19 using the ISARIC WHO Clinical Characterisation Protocol: prospective observational cohort study. BMJ 2020;369:m1985.

5 Keeley MP. Family communication at the end of life. Behav Sci 2017:7:45.

6 Samuel T, Beck K, Symonds R. Video consultations in UK primary care in response to COVID-19 pandemic. Br J Gen Pract 2020;70: 228-9.
7 Royal College of Physicians. Outpatients: the future - adding value through sustainability. London: RCP, 2018.

8 Greenhalgh T, Wherton J, Shaw S, Morrison C. Video consultations for covid-19. BMJ 2020;368:m998.

9 Silveira FJF da, Botelho CC, Valadao CCi. Breaking bad news: doctors' skills in communicating with patients. Sao Paulo Med J 2017;135:323-31.

10 Choe EK, Duarte ME, Suh H, Pratt W, Kientz JA. Communicating bad news: insights for the design of consumer health technologies. JMIR Hum Factors 2019;6:8885

11 Caswell G, Pollock K, Harwood R, Porock D. Communication between family carers and health professionals about end-of-life care for older people in the acute hospital setting: a qualitative study. BMC Palliat Care 2015;14:35

12 National Healthcare Communication Programme. Communication guide for difficult conversations. Health Service Executive, 2020. www.hse.ie/eng/about/our-health-service/healthcare-communication/nhcp-guidance-for-difficult-conversations.pdf

13 Field-Smith A, Robinson L. Talking to relatives: A guide to compassionate phone communication during COVID-19. NHS, 2020. www. bgs.org.uk/resources/covid-19-tools-and-templates\#\&gid=1\&pid = 1

14 Otani H, Yoshida S, Morita T et al. Meaningful communication before death, but not present at the time of death itself, is associated with better outcomes on measures of depression and complicated grief among bereaved family members of cancer patients. J Pain Symptom Manag 2017;54:273-9.

Address for correspondence: Dr Harriet L White, University Hospital Llandough, Penlan Road, Llandough, Penarth CF64 2XX, UK.

Email: hattiewhite94@gmail.com 